\begin{tabular}{|c|c|}
\hline \multirow{3}{*}{ 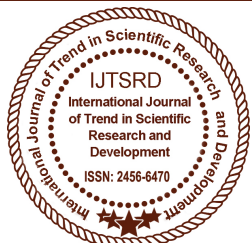 } & International Journal of Trend in Scientific Research and Development (IJTSRD) \\
\hline & International Open Access Journal | www.ijtsrd.com \\
\hline & ISSN No: 2456 - 6470 | Volume - 2 | Issue - 6 | Sep-Oct 2018 \\
\hline
\end{tabular}

\title{
Experimental Study on Properties of Clay Bricks by Adding Cement, Lime and SCBA
}

\author{
Singh Deepak Krishna ${ }^{1}$, Pratiksha Malviya ${ }^{2}$ \\ ${ }^{1}$ M.Tech Scholar, ${ }^{2}$ Professor \\ Department of Civil Engineering, Millennium Institute of Technology, \\ Bhopal, Madhya Pradesh, India
}

\section{INTRODUCTION}

Bricks are the artificial stones. These are used in 5. It can also be used to construct the boundary building construction and for ornamental purposes. These are one of the basic materials used for wall construction. These are easily available, cheap and light weighted. They can be mould into required shape and size. The properly manufactured bricks are nearly as strong as stone. walls.

\section{Types of Bricks}

Bricks can be of many types depending on -

1. Quality

2. Building Process

3. Manufacturing Method

Uses of Bricks

4. Raw Material

1. These bricks can be used as a building block to

5. Using Location construct the buildings.

6. Weather-resisting Capability

2. It can be used as a lining material for lining of 7 . Purpose of Using wells, canals etc.

8. Shape

3. It can also be used as a flooring material.

9. Region

4. It can also be used in Reinforced brick concrete.

\section{Literature Survey}

\begin{tabular}{|c|c|c|c|}
\hline $\begin{array}{c}\text { S } \\
\text { No }\end{array}$ & Authors & $\begin{array}{l}\text { Journal } \\
\& \text { Year }\end{array}$ & Brief Findings \\
\hline 1 & $\begin{array}{l}\text { Ravindra } \\
\text { Kumaret. } \\
\text { al }\end{array}$ & $\begin{array}{l}\text { IJERA } \\
2017\end{array}$ & $\begin{array}{l}\text { Burnt clay brick is one of the major and widely used building units in } \\
\text { construction around the world. The manufacturing of burnt clay bricks using } \\
\text { waste materials can minimize the environmental overburden caused by waste } \\
\text { deposition on open landfills and would also improve the brick performance } \\
\text { at low production cost leading to more sustainable construction. These } \\
\text { wastes utilization would not only be economical, but may also help to create } \\
\text { a sustainable and pollution free environment. This study aims to evaluate the } \\
\text { effect of the waste addition produced from two major crops: sugarcane and } \\
\text { rice in clay bricks manufacturing. }\end{array}$ \\
\hline 2 & $\begin{array}{l}\text { Saif A. } \\
\text { usmani }\end{array}$ & $\begin{array}{l}\text { IJESC } \\
2017\end{array}$ & $\begin{array}{l}\text { An attempt has been made to investigate the effect on mechanical properties } \\
\text { of bricks when blended with various wastes. Puzzolonic material along with } \\
\text { certain waste material blends will be studied, various mechanical properties } \\
\text { and their variation with change in composition is involved in the scope of } \\
\text { this study. Traditional brick-making would be the focus of research, the most } \\
\text { suitable material will be suggested based on the finding. }\end{array}$ \\
\hline
\end{tabular}


International Journal of Trend in Scientific Research and Development (IJTSRD) ISSN: 2456-6470

\section{Objectives}

Establishment of best suited combination of SCBA, lime, cement and other constituents proportion for higher compressive strength brick.

\section{Methodology}

The scope of current study aims at identifying nonconventional materials for use in bricks, this can be achieved by making trial mixes of various materials to be added in varying percentages. These bricks can then be fired using traditional methodologies. The physico-mechanical tests will be carried out on fired product according to recommended Indian standards. The tests are compressive strength IS 3495 (Part-I): 1992, water absorption IS 3495 (Part-II): 1992 efflorescence IS 3495 (Part-III): 1992 and brick density IS 2185 (PartI): 1979. The compressive strength will be determined using compression testing machine.

\section{RESULTS AND DISCUSSION}

Table 5.1 Specific Gravity of different materials those were used in manufacturing of bricks

\begin{tabular}{|c|c|c|}
\hline S. No. & Material & Specific Gravity \\
\hline 1 & SCBA & 2.28 \\
\hline 2 & Cement & 3.1 \\
\hline 3 & Lime & 2.4 \\
\hline
\end{tabular}

\section{Compressive strength analysis of the bricks}

The modular bricks samples of size $190 \mathrm{~mm} \times 90$ $\mathrm{mm} \times 90 \mathrm{~mm}$ (IS: 12894-2002) were casted. The compressive strength tests were conducted on three Bricks from each mix. The results of the compressive strength tests are shown in Table 5.2. These results were found after testing the specimens. Before testing, the frogs of the specimen were filled up with cement sand mortar $(1: 1)$.

Table 5.2 Compressive strength of Clay Brick

\begin{tabular}{|c|c|c|}
\hline $\begin{array}{c}\text { Specim } \\
\text { en No. }\end{array}$ & $\begin{array}{r}\text { Load taken by the } \\
\text { specimen brick (kg) }\end{array}$ & $\begin{array}{c}\text { Compressive } \\
\text { Strength } \\
\left(\mathrm{kg} / \mathrm{cm}^{2}\right)\end{array}$ \\
\hline 1 & 7700 & 45.03 \\
\hline 2 & 7800 & 45.61 \\
\hline 3 & 7750 & 45.32 \\
\hline
\end{tabular}

Average compressive strength of these specimen after testing $=45.32 \mathrm{~kg} / \mathrm{cm}^{2}$.
Table 5.3 Compressive strength of Brick Specimen (Mix-I)

\begin{tabular}{|c|c|c|c|}
\hline $\begin{array}{c}\text { Specim } \\
\text { en No. }\end{array}$ & $\begin{array}{l}\% \text { of } \\
\text { SCBA }\end{array}$ & $\begin{array}{l}\text { Load taken by } \\
\text { the specimen } \\
\text { brick (kg) }\end{array}$ & $\begin{array}{l}\text { Compressive } \\
\text { Strength } \\
\left(\mathrm{kg} / \mathrm{cm}^{2}\right)\end{array}$ \\
\hline 1 & 5 & 4702 & 27.47 \\
\hline 2 & 5 & 4653 & 27.18 \\
\hline 3 & 5 & 4711 & 27.48 \\
\hline
\end{tabular}

Average compressive strength of these specimen after testing $=27.38 \mathrm{~kg} / \mathrm{cm}^{2}$.

Table 5.4 Compressive strength of Brick Specimen (Mix-II)

\begin{tabular}{|c|c|c|c|}
\hline $\begin{array}{c}\text { Specim } \\
\text { en No. }\end{array}$ & $\begin{array}{c}\text { \% of } \\
\text { SCBA }\end{array}$ & $\begin{array}{c}\text { Load taken by } \\
\text { the specimen } \\
\text { brick (kg) }\end{array}$ & $\begin{array}{c}\text { Compressi } \\
\text { ve Strength } \\
\left(\mathrm{kg}^{2} \mathrm{~cm}^{2}\right)\end{array}$ \\
\hline 1 & 10 & 3802 & 22.21 \\
\hline 2 & 10 & 3852 & 22.50 \\
\hline 3 & 10 & 3854 & 22.52 \\
\hline
\end{tabular}

Average compressive strength of these specimen after testing $=22.41 \mathrm{~kg} / \mathrm{cm}^{2}$.

Table 5.5 Compressive strength of Brick Specimen (Mix-III)

\begin{tabular}{|c|c|c|c|}
\hline $\begin{array}{c}\text { Specim } \\
\text { en No. }\end{array}$ & $\begin{array}{c}\text { \% of } \\
\text { SCBA }\end{array}$ & $\begin{array}{c}\text { Load taken by } \\
\text { the specimen } \\
\text { brick (kg) }\end{array}$ & $\begin{array}{c}\text { Compressiv } \\
\text { e Strength } \\
\left(\mathrm{kg} / \mathrm{cm}^{2}\right)\end{array}$ \\
\hline 1 & 15 & 2602 & 15.21 \\
\hline 2 & 15 & 2651 & 15.48 \\
\hline 3 & 15 & 2552 & 14.92 \\
\hline
\end{tabular}

Average compressive strength of these specimen after testing $=15.20 \mathrm{~kg} / \mathrm{cm}^{2}$.

Table 5.6 Combine table for compressive Strength of Brick

\begin{tabular}{|c|c|c|c|}
\hline $\begin{array}{c}\text { Speci } \\
\text { men } \\
\text { No. }\end{array}$ & $\begin{array}{c}\text { \% of } \\
\text { SCBA }\end{array}$ & $\begin{array}{c}\text { Load taken by } \\
\text { the specimen } \\
\text { brick }(\mathrm{kg})\end{array}$ & $\begin{array}{c}\text { Compressive } \\
\text { Strength } \\
\left(\mathrm{kg} / \mathrm{cm}^{2}\right)\end{array}$ \\
\hline 1 & 5 & 4687 & 27.43 \\
\hline 2 & 10 & 3832.2 & 22.27 \\
\hline 3 & 15 & 2597 & 15.25 \\
\hline
\end{tabular}

Figure5.2 Compressive strength v/s SCBA

Table 5.7 Compressive strength of Brick Specimen (Mix-IV)

\begin{tabular}{|c|c|c|c|}
\hline $\begin{array}{c}\text { Speci } \\
\text { men } \\
\text { No. }\end{array}$ & $\begin{array}{c}\text { \% of } \\
\text { Cemen } \\
\mathrm{t}\end{array}$ & $\begin{array}{c}\text { Load taken by } \\
\text { the specimen } \\
\text { brick (kg) }\end{array}$ & $\begin{array}{c}\text { Compressiv } \\
\text { e Strength } \\
\left(\mathbf{k g} / \mathrm{cm}^{2}\right)\end{array}$ \\
\hline 1 & 5 & 10252 & 59.94 \\
\hline 2 & 5 & 10202 & 59.64 \\
\hline 3 & 5 & 10301 & 60.23 \\
\hline
\end{tabular}

Average compressive strength of these specimen after testing $=59.94 \mathrm{~kg} / \mathrm{cm}^{2}$. 
International Journal of Trend in Scientific Research and Development (IJTSRD) ISSN: 2456-6470

Table 5.8 Compressive strength of Brick Specimen (Mix-V)

\begin{tabular}{|c|c|c|c|}
\hline $\begin{array}{c}\text { Speci } \\
\text { men } \\
\text { No. }\end{array}$ & $\begin{array}{c}\text { \% of } \\
\text { Ceme } \\
\text { nt }\end{array}$ & $\begin{array}{c}\text { Load taken by } \\
\text { the specimen } \\
\text { brick }(\mathrm{kg})\end{array}$ & $\begin{array}{c}\text { Compressiv } \\
\text { e Strength } \\
\left(\mathrm{kg} / \mathrm{cm}^{2}\right)\end{array}$ \\
\hline 1 & 10 & 12010 & 70.17 \\
\hline 2 & 10 & 12108 & 70.76 \\
\hline 3 & 10 & 12152 & 71.05 \\
\hline
\end{tabular}

Average compressive strength of these specimen after testing $=70.62 \mathrm{~kg} / \mathrm{cm}^{2}$.

Table 5.9 Compressive strength of Brick Specimen (Mix-VI)

\begin{tabular}{|c|c|c|c|}
\hline $\begin{array}{c}\text { Speci } \\
\text { men } \\
\text { No. }\end{array}$ & $\begin{array}{c}\text { \% of } \\
\text { Cement }\end{array}$ & $\begin{array}{c}\text { Load taken by } \\
\text { the specimen } \\
\text { brick (kg) }\end{array}$ & $\begin{array}{c}\text { Compressi } \\
\text { ve Strength } \\
\left(\mathrm{kg} / \mathrm{cm}^{2}\right)\end{array}$ \\
\hline 1 & 15 & 18002 & 105.26 \\
\hline 2 & 15 & 17002 & 99.42 \\
\hline 3 & 15 & 17251 & 100.88 \\
\hline
\end{tabular}

Average compressive strength of these specimen after testing $=101.86 \mathrm{~kg} / \mathrm{cm}^{2}$.

Table 5.10 Combine table for compressive Strength of Brick

\begin{tabular}{|c|c|c|c|}
\hline $\begin{array}{c}\text { Speci } \\
\text { men } \\
\text { No. }\end{array}$ & $\begin{array}{c}\text { \% of } \\
\text { Cement }\end{array}$ & $\begin{array}{c}\text { Load taken by } \\
\text { the specimen } \\
\text { brick }(\mathrm{kg})\end{array}$ & $\begin{array}{c}\text { Compressi } \\
\text { ve Strength } \\
\left(\mathrm{kg} / \mathrm{cm}^{2}\right)\end{array}$ \\
\hline 1 & 5 & $10248 . .2$ & 59.93 \\
\hline 2 & 10 & 12072.7 & 70.06 \\
\hline 3 & 15 & 17416.4 & 101.85 \\
\hline
\end{tabular}

Figure5.3 Compressive strength v/s Cement

Table 5.11 Compressive strength of Brick Specimen (Mix-VII)

\begin{tabular}{|c|c|c|c|}
\hline $\begin{array}{c}\text { Speci } \\
\text { men } \\
\text { No. }\end{array}$ & $\begin{array}{c}\text { \% of } \\
\text { Lime }\end{array}$ & $\begin{array}{c}\text { Load taken by } \\
\text { the specimen } \\
\text { brick }(\mathrm{kg})\end{array}$ & $\begin{array}{c}\text { Compressiv } \\
\text { e Strength } \\
\left(\mathrm{kg} / \mathrm{cm}^{2}\right)\end{array}$ \\
\hline 1 & 5 & 8000 & 46.78 \\
\hline 2 & 5 & 8100 & 47.37 \\
\hline 3 & 5 & 8150 & 47.66 \\
\hline
\end{tabular}

Average compressive strength of these specimen after testing $=47.28 \mathrm{~kg} / \mathrm{cm}^{2}$.

Table 5.12 Compressive strength of Brick Specimen (Mix-VIII)

\begin{tabular}{|c|c|c|c|}
\hline $\begin{array}{c}\text { Specim } \\
\text { en No. }\end{array}$ & $\begin{array}{c}\text { \% of } \\
\text { Lime }\end{array}$ & $\begin{array}{c}\text { Load taken by } \\
\text { the specimen } \\
\text { brick (kg) }\end{array}$ & $\begin{array}{c}\text { Compressive } \\
\text { Strength } \\
\left(\mathrm{kg} / \mathrm{cm}^{2}\right)\end{array}$ \\
\hline 1 & 10 & 10100 & 59.06 \\
\hline 2 & 10 & 10000 & 58.47 \\
\hline 3 & 10 & 10200 & 59.65 \\
\hline
\end{tabular}

Average compressive strength of these specimen after testing $=59.07 \mathrm{~kg} / \mathrm{cm}^{2}$.
Table 5.13 Compressive strength of Brick Specimen (Mix-IX)

\begin{tabular}{|c|c|c|c|}
\hline $\begin{array}{c}\text { Speci } \\
\text { men } \\
\text { No. }\end{array}$ & $\begin{array}{c}\% \text { of } \\
\text { Lime }\end{array}$ & $\begin{array}{c}\text { Load taken by } \\
\text { the specimen } \\
\text { brick }(\mathrm{kg})\end{array}$ & $\begin{array}{c}\text { Compressi } \\
\text { ve Strength } \\
\left(\mathrm{kg} / \mathrm{cm}^{2}\right)\end{array}$ \\
\hline 1 & 15 & 13002 & 76.02 \\
\hline 2 & 15 & 13105 & 76.61 \\
\hline 3 & 15 & 12854 & 75.15 \\
\hline
\end{tabular}

Average compressive strength of these specimen after testing $=75.95 \mathrm{~kg} / \mathrm{cm}^{2}$.

Table 5.14 Combine table for compressive Strength of Brick

\begin{tabular}{|c|c|c|c|}
\hline $\begin{array}{c}\text { Specim } \\
\text { en No. }\end{array}$ & $\begin{array}{c}\text { \% of } \\
\text { Lime }\end{array}$ & $\begin{array}{c}\text { Load taken by } \\
\text { the specimen } \\
\text { brick (kg) }\end{array}$ & $\begin{array}{c}\text { Compressiv } \\
\text { e Strength } \\
\left(\mathrm{kg} / \mathrm{cm}^{2}\right)\end{array}$ \\
\hline 1 & 5 & 8083.9 & 47.27 \\
\hline 2 & 10 & 10099.25 & 59.06 \\
\hline 3 & 15 & 12984.02 & 75.95 \\
\hline
\end{tabular}

\section{CONCLUSION}

On the basis of observation the following conclusions are:

1- Increase the compressive strength of bricks around $60-64 \%$ with use of $15 \%$ lime in manufacturing of bricks.

2- Increase the compressive strength of bricks around 120 - $125 \%$ with use of $15 \%$ cement in manufacturing of bricks.

3- If add SCBA in the mix, it reduces the compressive strength of brick.

\section{REFERENCES}

1. ASTM (1985). "ASTM standard test method for unconfined compressive strength of soil." ASTM D2166, Philadelphia.

2. Dhir, R.K. (2005): "Emerging trends in fly ash utilization: World Scenario", Proc. Of International Conference on fly ash utilization, pp: O 1.1-1.10.

3. Fibre Reinforced Fly Ash Based Lime Bricks", International Journal of the IS: 12894-2002, Pulverized Fuel Ash-Lime Bricks-Specification, Bureau of Indian Standards, New Delhi.

4. IS: 3495 (Part 1 and 2)-1992, Methods of tests of Burnt Clay Building Bricks- Specification, Bureau of Indian Standards, New Delhi.

5. IS: 6932-1973, Methods of tests for building lime-Specification, Bureau of Indian Standards, New Delhi. 
6. J. N. Akhtar, J. Alam and M. N. Akhtar (2010), "An Experimental Study on Kumar Virender, (May 2004), Compaction and Permeability Study of Pond Ash Amended with Locally Available Soil and Hardening Agent, http://www.ieindia.org/publish/cv/0504/may04cv5 .pdf.

7. Lee W. E., Souzza G.P., McConville C. J., Tarvompanich T. \& Yqbal Y. (2008) Mullite formation in clays and clay-derived vitreous ceramics. Journal of the Europen ceramic Society 28/2, 465-471.

8. Matrai J. (1977) A POROTON teglak gyartastechnologiajaroles jellemzoirol. 19/6, 246-252.
9. Mindess, S., Young, J. F., and Darwin, D. (2004). "Aggregates and Response of Concrete to Stress" Concrete, 2nd Ed., Pearson Education, Inc. Upper Saddle River, NJ, Chap. 7, 121, and Chap. 13, 318.

10. Mishra Manoj Kumar, (December 2006) Geotechnical Characterization of Fly Ash.

11. N. S. Pandian and S. Balasubramonian, Leaching studies on ASTM type F fly ashes by an accelerated process method, J. Testing Evaluation, ASTM, 28, 44-51 (2000).

12. 12. Palagyi G. (1976) A tegla multjabol Epitoanyag, 27/7, 269-274.

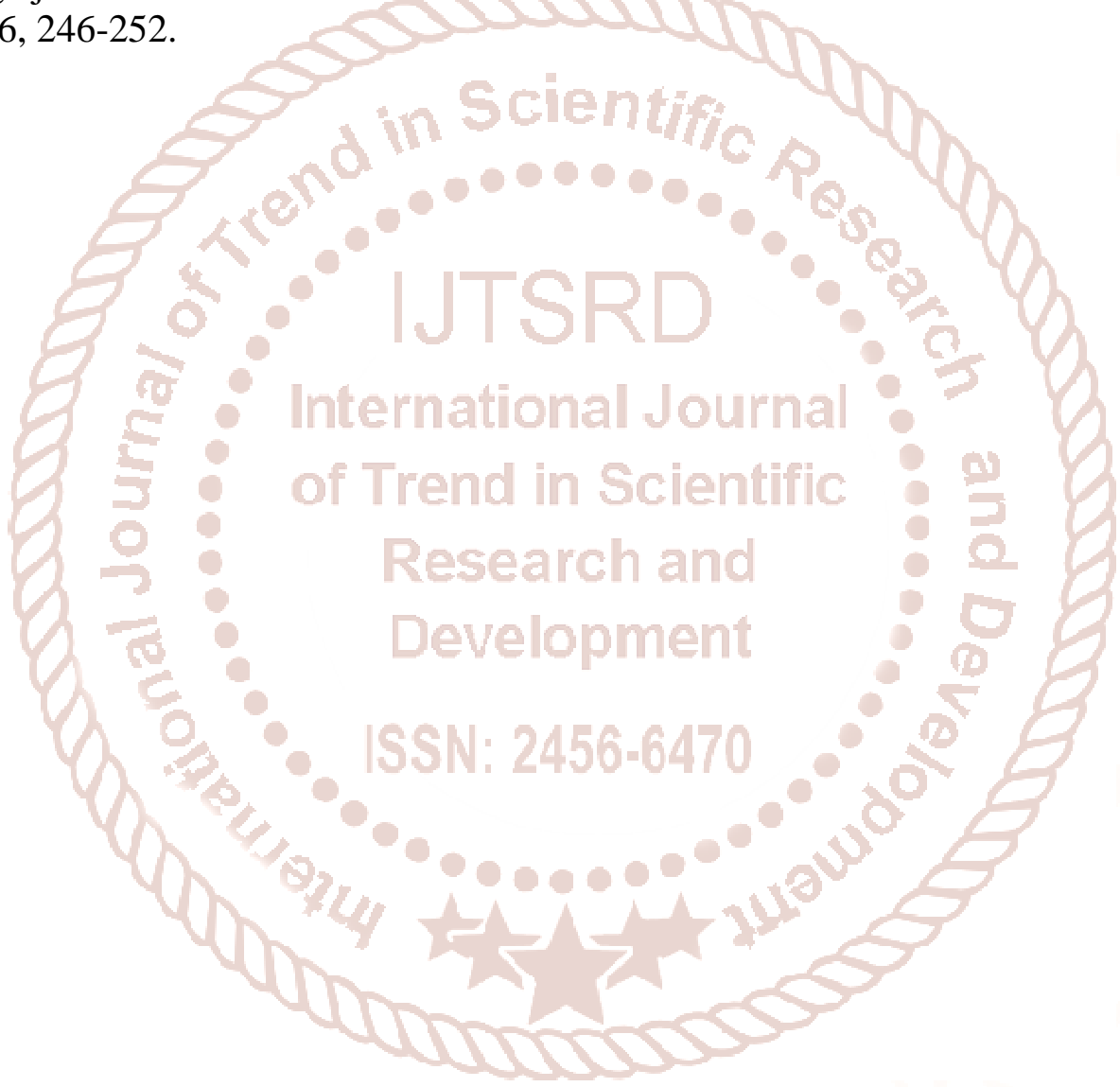

